\title{
CLASSIFICATION OF SOLUTIONS OF DELAY DIFFERENCE EQUATIONS
}

\author{
B.G. ZHANG \\ Department of Applied Mathematics \\ Ocean University of Qingdao \\ Qingdao, China 266003 \\ and \\ PENGXIANG YAN \\ Department of Mathematics \\ University of Alberta \\ Edmonton, Alberta, Canada T6G 2G1
}

(Received August 20, 1992 and in revised form November 1, 1992)

ABSTRACT. In this paper we study the classification of solutions of delay difference equation

$$
\left\{\begin{array}{l}
\Delta^{2} y_{n}=P_{n} y_{n-m} \\
y_{n}=A_{n} \text { for } n=N-(m+1), \cdots N-1
\end{array}\right.
$$

where $A_{n}, n=N-(m+1), \cdots, N-1$ are given, $m$ is a nonnegative integer.

KEY WORDS AND PHRASES. Delay difference equations, oscillation, bounded solutions.

1991 AMS SUBJECT CLASSIFICATION CODES. Primary, 39A10.

1. INTRODUCTION. The problem of oscillation and nonoscillation of solutions of delay difference equations has been receiving a lot of attention for the last few years. Erbe and Zhang ([1]-[3]), Lalli, Zhang and Zhao ([8], [9]), Ladas, Philos and Sficas ([6], [7]), have done some extensive works on this topic. A survey on the oscillation of delay difference equations could be found in the monograph by Gyori and Ladas [5].

In this paper we consider the second order delay difference equations of the form:

$$
\Delta^{2} y_{n}=P_{n} y_{n-m}
$$

where $\Delta$ denotes the forward difference operator: $\Delta y_{n}=y_{n+1}-y_{n}, m$ is a nonnegative integer.

By a solution of equation (1.1) we mean a sequence $\left\{y_{n}\right\}$ which is defined for $n \geq N-(m+1)$ and which satisfies equations (1.1) for all $n \geq N$. Clearly if

$$
y_{n}=A_{n}, \text { for } n=N-(m+1), N-m, \cdots, N
$$

are given, then equation (1.1) has a unique solution satisfying the initial conditions (1.2), where $N$ is an initial point.

A nontrivial solution $\left\{y_{n}\right\}$ of equation (1.1) is said to be oscillatory if for every $N>0$ there exists an $n \geq N$ such that $y_{n} y_{n+1} \leq 0$. Otherwise it is called nonoscillatory.

Set $E_{N}=\{N-(m+1), N-m, \cdots, N-1\}$, if

$$
y_{n}=A_{n}, n \in E_{N}
$$


are given, then the solutions depend on the parameter $y_{N}=\xi$. We are concerning with the classification of solutions of equation (1.1) with (1.3).

\section{MAIN RESULTS.}

We always assume that $P_{n} \geq 0$ and $P_{n}$ does not identically equal to zero in equation (1.1). We denote $S$ the set of all solutions of (1.1). Since $P_{n} \geq 0$, it is easy to see that

$$
S=s^{+\infty} \bigcup s^{-\infty} \bigcup s^{k} \bigcup S^{-k} \bigcup S^{o} \bigcup S^{\sim}
$$

where

$$
\begin{aligned}
S^{+\infty} & =\left\{\left\{y_{n}\right\} \in S: \lim _{n \rightarrow \infty} y_{n}=+\infty\right\} \\
S^{-\infty} & =\left\{\left\{y_{n}\right\} \in S: \lim _{r \rightarrow \infty} y_{n}=-\infty\right\} \\
S^{k} & =\left\{\left\{y_{n}\right\} \in S: 0<\lim _{n \rightarrow \infty} y_{n}<+\infty\right\} \\
S^{-k} & =\left\{\left\{y_{n}\right\} \in S: 0>\lim _{n \rightarrow \infty} y_{n}>-\infty\right\} \\
S^{o} & =\left\{\left\{y_{n}\right\} \in S: y_{n} \text { nontrivial, } \lim _{n \rightarrow \infty} y_{n}=0 \text { monotonically }\right\} \\
S^{\sim} & =\left\{\left\{y_{n}\right\} \in S: y_{n} \text { is oscillatory }\right\} .
\end{aligned}
$$

LEMMA 2.1 If

$$
y_{1} \geq 0 \text { on } E_{N}, y_{N}>y_{N-1}
$$

then $y_{n} \in S^{+\infty}$. If

$$
y_{\imath} \leq 0 \text { on } E_{N}, y_{N}<y_{N-1}
$$

than $y_{n} \in S^{-\infty}$.

PROOF. From (1.1), we have

$$
\Delta y_{N+n}-\Delta y_{N-1}=\sum_{i=N-1}^{N+(n-1)} P_{i} y_{i-m},
$$

Summing it in $n$ we have

$$
y_{N+n}=y_{N-1}+n \Delta y_{N-1}+\sum_{i=0}^{n-1} \sum_{j=N-1}^{N+n-1} P_{j} y_{j-m} .
$$

The conclusions of Lemma 2.1 follow from (2.2).

From (2.2), the following is also true.

LEMMA 2.2. If

then

$$
\lim _{n \rightarrow \infty} \sum_{i=N-1}^{n+N-2}(n+N-1-i) P_{i}=\infty
$$

$$
y_{i} \geq 0, i \in E_{N}, y_{N} \geq y_{N-1}
$$

imply that $\left\{y_{n}\right\} \in S^{+\infty}$, and if

$$
y_{i} \leq 0, i \in E_{N}, y_{N} \leq y_{N-1}
$$

imply that $\left\{y_{n}\right\} \in S^{-\infty}$.

LEMMA 2.3. Assume that the solution $y_{n}$ and $z_{n}$ have same initial values on $E_{N}$ with $\Delta y_{N-1}>\Delta z_{N-1}$. Then $y_{n}>z_{n}, \Delta y_{n}>\Delta z_{n}, n \geq N$ and

$$
\lim _{n \rightarrow \infty}\left(y_{n}-z_{n}\right)=\infty
$$


PROOF. Set $x_{n}=y_{n}-z_{n}$, then $x_{1}=0$ on $E_{N}$ and $\Delta x_{N-1}>0$. By Lemma 2.1, $\left\{x_{n}\right\} \in S^{+\infty}$ From (2.1) $\Delta x_{n}>0$ for $n \geq N$.

LEMMA 2.4. For every given initial value on $E_{N}$. equation (1.1) has no more than one bounded solution.

PROOF. Suppose the contrary, let $\left\{y_{n}\right\},\left\{z_{n}\right\}$ be two bounded solutions of (1.1) with $y_{1}=z_{\text {, }}$ on $E_{N}$ and $y_{N}>z_{N}$. This implies that $\left|y_{n}-z_{n}\right|$ is bounded. On the other hand, by Lemma 2.3, (2.4) should be true. This contradiction proves Lemma 2.4 .

For given $y_{\mathrm{t}}=A_{\mathrm{t}}$ on $E_{N}$, then the solution of (1.1) depends on the parameter $y_{N}=\xi \in R$. Define the sets of $\xi$ as follows:

$$
\begin{aligned}
K^{+\infty} & =\left\{\xi \in R,\left\{y_{n}\right\} \in S^{+\infty}\right\} \\
K^{-\infty} & =\left\{\xi \in R,\left\{y_{n}\right\} \in S^{-\infty}\right\} \\
K^{o} & =\left\{\xi \in R,\left\{y_{n}\right\} \in S^{o}\right\} \\
K^{\sim} & =\left\{\xi \in R,\left\{y_{n}\right\} \in S^{\sim}\right\}
\end{aligned}
$$

THEOREM 2.1. For given $y_{\mathrm{a}}$ on $E_{N}$, the sets $K^{+\infty}$ and $K^{-\infty}$ are nonempty.

PROOF. If $y_{1}=0$ on $E_{N}$, the conclusion follows from Lemma 2.1. Otherwise, from (2.1) and (2.2) we can find a number $y_{N}=\xi$ so large that $y_{\imath}>0, i=N, N+1, \cdots N+m$ and $\Delta y_{N+m}>0$. Translating the initial point to $n+m$ and using Lemma 2.1 we conclude that the solution with this $y_{N}$ belongs to $S^{+\infty}$. Therefore $\xi \in K^{+\infty}$. It is similar to prove that $K^{-\infty}$ is nonempty.

THEOREM 2.2. The sets $K^{-\infty}, K^{+\infty}$ are open sets which are given by nonintersecting half lines $(-\infty, \alpha)$ and $(\beta,+\infty)(\alpha \leq \beta)$. The set $F=R-\left(K^{+\infty} \cup K^{-\infty}\right)$ is nonempty and consists of the interval $[\alpha, \beta]$, if $\alpha<\beta$, or the point $\alpha$, if $\alpha=\beta$.

PROOF. Let $\left\{y_{n}\right\} \in S^{+\infty}$. Then there exists $N^{\prime}$ such that $y_{\mathrm{v}}>0$ and $\Delta y_{\mathrm{v}}>0$ on $E_{N^{\prime \prime}}$ By continuous dependence of solutions and their differences on the initial conditions, all solutions with $y_{1}$ on $E_{N}$ and $\bar{y}_{N}$ differ slightly from $y_{N}$ are positive and have positive differences on $E_{N^{\prime \prime}}$. If the initial point is translated to the point $i=N^{\prime}$, then by Lemma 2.1 all those solutions belong to $S^{+\infty}$, i.e., $K^{+\infty}$ is open. Similarly, one can prove that $K^{-\infty}$ is open. Using Lemma 2.3 , the conclusions of theorem follow.

THEOREM 2.3. If $\alpha<\beta$, then each $y_{N} \in F$ the corresponding solution is unbounded and oscillatory.

PROOF. It is sufficient to show that every solution with $y_{N} \in F$ is unbounded. Suppose the contrary, $\left\{y_{n}\right\}$ is a bounded solution with $y_{N} \in F$. Let $z_{N} \neq y_{N}$. By Lemma 2.4, $\left\{z_{n}\right\}$ is unbounded and oscillatory. On the other hand, Lemma 2.3 shows that $\left|y_{n}-z_{n}\right| \rightarrow \infty$ as $n \rightarrow \infty$ and hence $\lim _{n \rightarrow \infty}\left|z_{n}\right|=\infty$ which contradicts the oscillation of $\left\{z_{n}\right\}$.

THEOREM 2.4. If $\sum_{i=N}^{\infty} i P_{\imath}=\infty$, then every bounded solution of (1.1) either belongs to $S^{o}$ or $S^{\sim}$.

PROOF. Let $\left\{y_{n}\right\}$ be a bounded positive solution of (1.1). Then

From (2.1)

$$
\Delta y_{n}<0 \text { eventually and } \lim _{n \rightarrow \infty} \Delta y_{n}=0
$$

$\Delta y_{N-1}=-\sum_{i=N-1}^{\infty} P_{i} y_{t-m}$

and from (2.2) 


$$
\begin{aligned}
& y_{N+n}=y_{N-1}-n \sum_{i=N-1}^{\infty} P_{\imath} y_{\imath-m}+\sum_{i=0}^{n-1} \sum_{j=N-1}^{N+i-1} P_{j} y_{j-m} \\
& =y_{N-1}-n \sum_{i=N-1}^{\infty} P_{\imath} y_{i-m}+\sum_{i=N-1}^{N+n-2}(n+N-1-i) P_{\imath} y_{i-m} \\
& =y_{N-1}-n \sum_{i=N-1}^{N+n-2} P_{i} y_{1-m}-n \sum_{i=N+n-1}^{\infty} P_{i} y_{i}-m+\sum_{i=N-1}^{N+n-2}(n+N-1-i) P_{i} y_{i}-m \\
& =y_{N-1}-n \sum_{i=N+n-1}^{\infty} P_{i} y_{i}-m+\sum_{i=N-1}^{N+n-2}(N-1-i) P_{1} y_{i}-m \\
& =y_{N-1}-n \sum_{i=N+n-1}^{\infty} P_{i} y_{i-m}+(N-1) \sum_{i=N-1}^{N+n-2} P_{i} y_{i-m}-\sum_{i=N-1}^{N+n-2} i P_{i} y_{i-m} \\
& =y_{N-1}+(N-1)\left(\Delta y_{N+n-2}-\Delta y_{N-1}\right)-\sum_{i=N-1}^{N+n-2} i p_{\imath} y_{t-m}+n \Delta y_{N+n-1} \\
& \leq y_{N-1}-(N-1) \Delta y_{N-1}-\sum_{i=N-1}^{N+n-2} i p_{i} y_{i-m} .
\end{aligned}
$$

If $y_{n} \rightarrow l>0$, then (2.5) lead to that $\lim _{n \rightarrow \infty} y_{n}=-\infty$. This contradiction shows that $\lim _{n \rightarrow \infty} y_{n}=0$. The proof is complete.

COROLLARY 2.1. If $\sum_{i=N}^{\infty} i p_{t}=\infty$, then

$$
R=K^{+\infty} \bigcup K^{-\infty} \bigcup K^{\circ} \bigcup K^{\sim}
$$

and $K^{+\infty}, K^{-\infty}$ and $K^{\circ} \cup K^{\sim}$ are nonempty.

THEOREM 2.5. Assume that

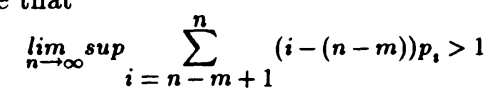

Then every bounded solution of (1.1) is oscillatory.

PROOF. Let $\left\{y_{n}\right\}$ be a bounded positive solution of (1.1). Then $\Delta y_{n}<0$ eventually. Summing (1.1) from $N$ to $n$, we have

$$
\Delta y_{n+1}-\Delta y_{N}=\sum_{i=N}^{n} p_{i} y_{i-m}
$$

Summing it from $n-m+1$ to $n$ in $N$, we obtain

Hence

$$
m \Delta y_{n+1}-y_{n+1}+y_{n-m+1}=\sum_{j=n-m+1}^{n} \sum_{i=j}^{n} p_{i} y_{i-m}
$$

$$
\begin{aligned}
0 & \leq y_{n+1}-y_{n-m+1}+\sum_{i=n-m+1}^{n}(i-(n-m)) p_{i} y_{i}-m \\
& \leq y_{n+1}-y_{n-m+1}\left(1-\sum_{i=n-m+1}^{n}(i-(n-m)) p_{i}\right)
\end{aligned}
$$

which contradicts to (2.7). The proof is complete.

COROLLARY 2.2 Assume that the assumptions of Corollary 2.1 and Theorem 2.5 hold. 
Then $K^{\sim} \sim$ is nonempty.

In fact, by Corollary $2.1, K^{\circ} \cup K^{\sim}$ is nonempty and by Theorem $2.5, K^{\circ}$ is empty Therefore $\boldsymbol{h}^{\sim}$ is nonempty.

It is easy to see that if $p_{1} \equiv p>0$ in (1.1), then all assumptions of Corollary 2.2 hold, therefore for any given $A_{n}$ on $E_{N}$, equation (1.1) with (1.3) has at least one oscillatory solution, i.e., $K \sim$ is nonempty.

EXAMPLE 2.1. Consider

$$
\Delta^{2} y_{n}=P_{n} y_{n-4}
$$

with $y_{1}=(-1)^{2}, \imath=1, \cdots, 5 P_{n} \equiv 1$. Then through computation if $y_{6}>-0.21675$, the solution $\left\{y_{n}\right\} \in S^{+\infty}$, if $y_{6}<-0.21676$, the solution $\left\{y_{n}\right\} \in S^{-\infty}$, in this case we see $\alpha=\beta$.

OPEN PROBLEM. What condition could guarantee that $\alpha<\beta$ ?

ACKNOWLEDGEMENT. This research paper was supported by NSERC-Canada and was carried out while visiting the University of Alberta.

\section{REFERENCES}

1. ERBE, L.H. \& ZHANG, B.G., Oscillation of difference equations with delay, Proceeding of the Internationa; Conference on Theory and Applications of Differential Equations. Ohio University (1988), 257-263; Editor, A.R. Aftabijadeh.

2. ERBE, L.H. \& ZHANG, B.G., Oscillation of second order linear difference equations, Chinese Mathematical Journal 16 (4) (1988), 239-252.

3. ERBE, L.H. \& ZHANG, B.G., Oscillation of discrete analogue of delay equations, Diff. Int Equations 2 (1989), 300-309.

4. GEDRGIOUS, D.A.; GROVE, E.A. \& LADAS, G., Oscillation of neutral difference equations, Appl. Anal. $\underline{33}(34)$ (1989), 243-253.

5. GYORI, I. \& LADAS, G., Oscillation Theory of Delay Differential Equations with Applications, Clarenden Press, Oxford, 1991.

6. LADAS, G., Recent developments in the oscillation of delay difference equations, Differential Equations: Stability and Control (1990), 321-332; Editor Marcel Dekker.

7. LADAS, G.; PHILOS, C.G. \& SFICAS, Y.G., Necessary and sufficient conditions for the oscillation of difference equations, Liberta $\underline{\text { Math }} \underline{9}$ (1989), 121-125.

8. LALLI, B.S. \& ZHANG, B.G., On existence of positive solutions and bounded oscillation for neutral difference equations, J. Math. Anal. Appl. 166 (1) (1992), 272-287.

9. LALLI, B.S.; ZHANG, B.G. \& ZHAO, L.J., On oscillation and existence of positive solutions of neutral difference equations, J. Math. Anal. Appl. 158 (1) (1991), 213-233. 


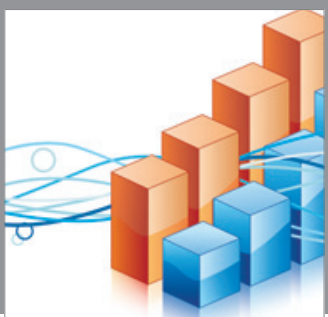

Advances in

Operations Research

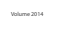

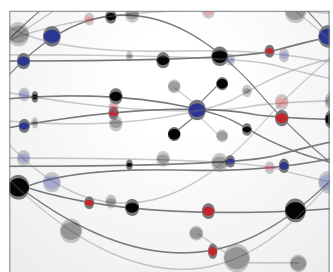

\section{The Scientific} World Journal
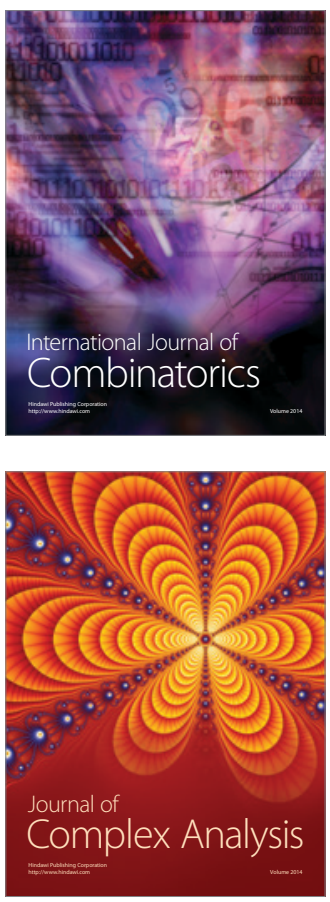

International Journal of

Mathematics and

Mathematical

Sciences
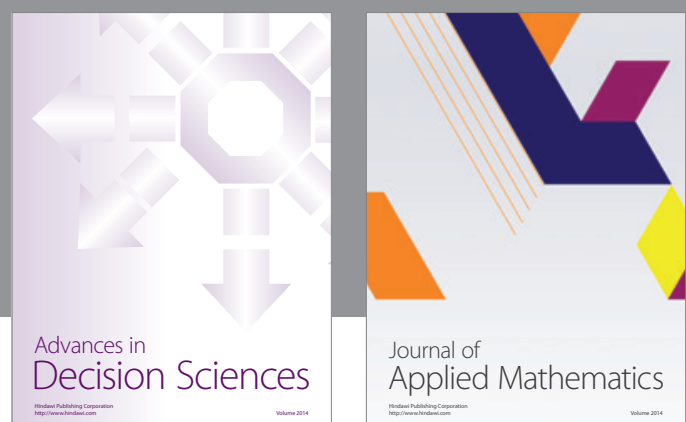

Journal of

Applied Mathematics
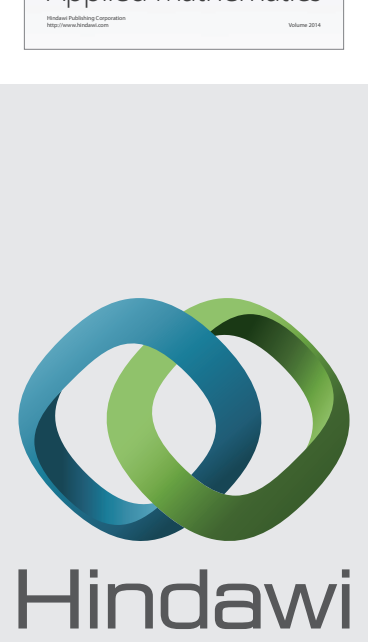

Submit your manuscripts at http://www.hindawi.com
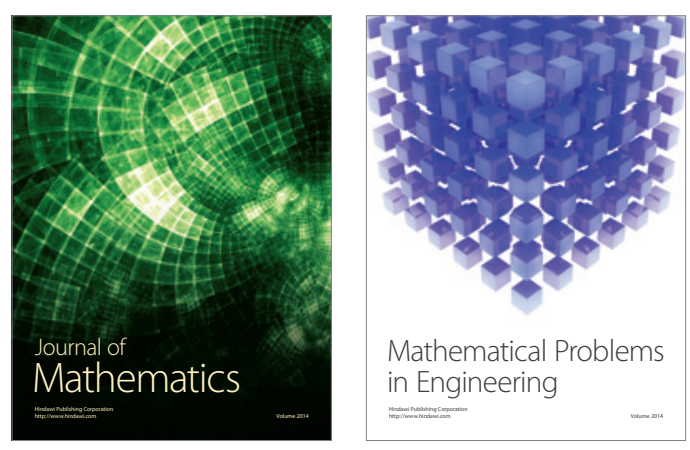

Mathematical Problems in Engineering
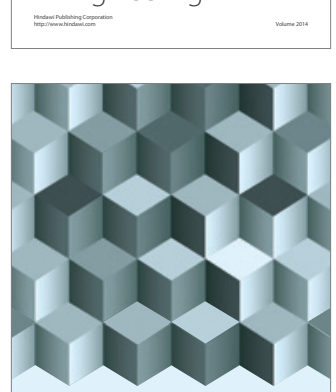

Journal of

Function Spaces
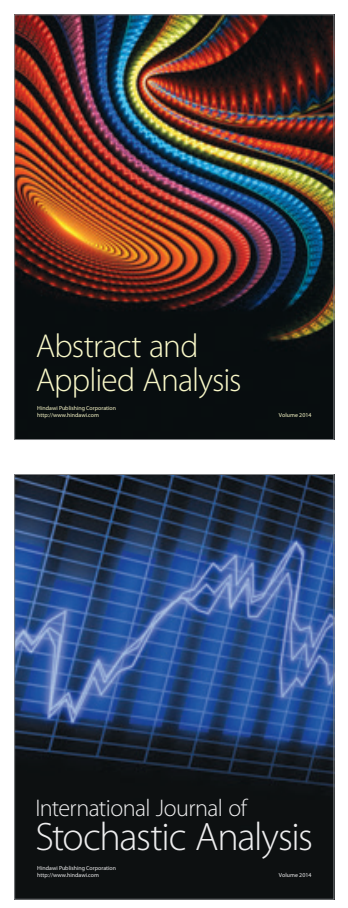

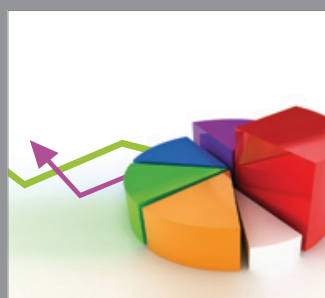

ournal of

Probability and Statistics

Promensencen
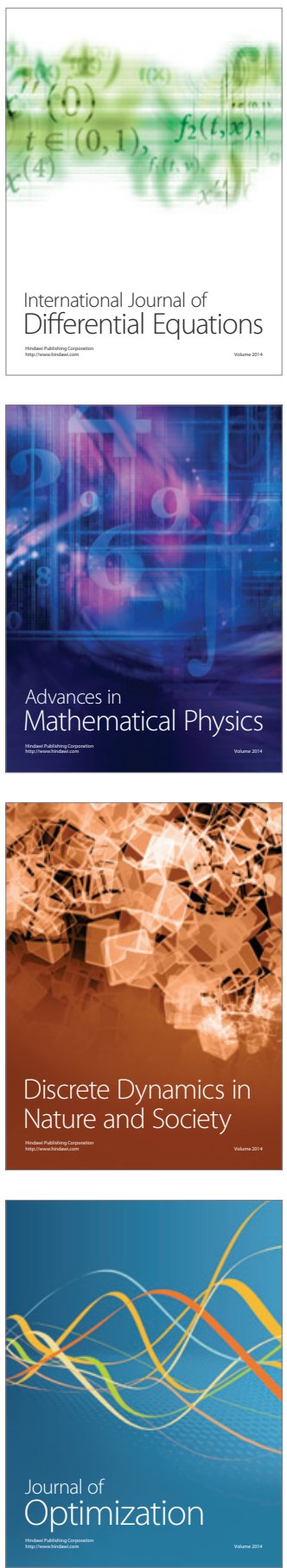